\title{
RESISTENCIA A SEQUÍA TERMINAL EN FRIJOL NEGRO TROPICAL1
}

\author{
Mark Frahm ${ }^{2}$, Juan Carlos Rosas ${ }^{3}$, Netzahualcoyotl Mayek ${ }^{4}$, Ernesto López ${ }^{5}$, Jorge A. Acosta ${ }^{6}$, \\ James D. Kelly ${ }^{2}$
}

\section{RESUMEN}

Resistencia a sequía terminal en frijol negro tropical. Con el objetivo de identificar cultivares de frijol negro tropical con resistencia a sequía y adaptación a la región Centroamericana, se diseñó un proyecto piloto colaborativo entre los programas de frijol de Zamorano, Honduras; Veracruz, México y la Universidad Estatal de Michigan (MSU); EE.UU, en el año 2002. Se desarrollaron dos poblaciones de líneas endogámicas recombinantes (RILs) derivadas de cruces entre la línea resistente a sequía B98311 de MSU con TLP 19 y VAX 5, dos líneas mejoradas del CIAT con resistencia a enfermedades y adaptadas a las condiciones de América Central y México. Ambas poblaciones se evaluaron bajo riego y sequía terminal en Zamorano, Honduras y Veracruz, México. Los rendimientos se vieron reducidos por la sequía y por Macrophomina phaseolina, hongo causal de la pudrición carbonosa. El estrés por sequía, la presión de la enfermedad y los bajos rendimientos, contribuyeron a la obtención de altos coeficientes de variación, lo que hizo difícil la identificación y selección de líneas superiores. La selección se basó en la posición de la media geométrica del rendimiento $(\mathrm{MG})$ calculada para cada línea con los datos de ambos tratamientos, riego y sequía. La línea L88-63 ocupó el primer lugar en la MG del rendimiento en ambas localidades. Evaluaciones posteriores conducidas en Honduras y Michigan, confirmaron el alto potencial de rendimiento y amplia adaptación de la línea L8863. El mejoramiento de la resistencia a la sequía en ambientes del trópico bajo, requiere la incorporación adicional de resistencia a $M$. phaseolina en las nuevas líneas consideradas para liberarse como nuevas variedades en esta región.

\begin{abstract}
Resistance of tropical black beans to terminal drought. A pioneer collaboration between the breeding programs in Honduras, Mexico and Michigan State University (MSU) was designed to identify commercial black bean cultivars exhibiting drought resistance adapted for Central America and regions in Mexico. Two recombinant inbred line (RIL) populations were developed from crosses between a drought resistant line, B98311 from MSU, with TLP 19 and VAX 5, two lines from CIAT with improved disease resistance and adapted to the growing conditions in Latin America. Both populations were tested (in Zamorano, Honduras and Veracruz, Mexico) under drought stress and non-stress conditions. Yields were reduced by drought stress and Macrophomina phaseolina, the causal fungal pathogen of charcoal rot. Drought stress, disease pressure and low yields contributed to the high variation coefficients (VC), which hindered the identification and selection for superior lines. Selection was based on rank of the geometric mean (GM) yield, calculated from the yield of each line in the stress and non-stress treatments. The RIL, L88-63 ranked first in GM yield at both locations. Subsequent testing in Honduras and Michigan supported the high yield potential and broad adaptation of L88-63. Breeding for drought resistance in lowland tropical environments will require that additional resistance to $M$. phaseolina be incorporated into potential new bean lines being considered for release in this region.
\end{abstract}

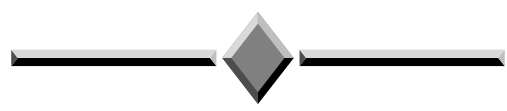

1 Recibido para publicación el 27 de abril del 2003. Presentado en la XLIX Reunión Anual del PCCMCA, La Ceiba, Honduras 2003.

2 Dept. of Crop and Soil Science, Michigan State Univ., E. Lansing, MI 48824.

3 EAP/Zamorano, P.O. Box 93, Tegucigalpa, Honduras.

4 Departamento de Química, Universidad Autónoma de Aguascalientes, Universidad 940, CP 20100, Aguascalientes, México.

5 INIFAP Campo Experimental Cotaxtla, Km 34 Carretera Veracruz-Córdoba, A. Postal 429, CP 91700, Veracruz, México

6 INIFAP Campo Experimental Bajío, Km 6.5 Carretera Celaya-San Miguel de Allende, A. Postal 112, CP 38110 Celaya, México. 


\section{INTRODUCCIÓN}

La sequía terminal es una limitante anual de la producción de frijol común (Phaseolus vulgaris L.) en América Central y México. En ambientes del trópico bajo, un patrón bimodal de lluvias permite dos épocas de producción. En la primera época (mayo a agosto) ocurre la mayor precipitación; mientras que, en la segunda (Postrera; septiembre a diciembre) la precipitación es limitada. Más del $60 \%$ del área cultivada con frijol en Honduras se siembra en postrera bajo el sistema de relevo después de maíz, Zea mays (Rosas et al. 1991). El corto ciclo biológico del frijol común lo convierte en el cultivo ideal para sembrarse al final de la primera época con humedad residual; sin embargo, la humedad disminuye durante este cultivo provocando sequía terminal. El área de producción de frijol en Honduras se incrementa tres veces durante la postrera en comparación con la primera, pero el rendimiento es del $50 \%$ debido a sequía terminal (Cotty et al. 2001). La utilización de irrigación es poco realista; por ello, el mejoramiento genético para resistencia de sequía ofrece una solución de largo plazo para la productividad del frijol bajo sequía en América Central y México.

La resistencia a sequía se define por el rendimiento relativo de un genotipo comparado con otros genotipos sujetos a la misma sequía (Hall 1993). El rendimiento se mide bajo condiciones de sequía (Rs) y riego $(\mathrm{Rr})$, y se calcula la media geométrica del rendimiento $(\mathrm{MG}=(\mathrm{Rr} * \mathrm{Rs})-)$ para los genotipos individuales. La MG ha mostrado ser el criterio de selección más efectivo para resistencia a sequía en frijol; para su cálculo, se necesitan datos de rendimiento, riego y sequía, y es una medida del potencial rendimiento de cada genotipo (Abebe et al., 1998; Samper 1984). Para una selección efectiva de resistencia a sequía en frijol común, se ha propuesto basar la selección en una alta MG, seguida por selección para alto rendimiento bajo sequía (Schneider et al. 1997).

El germoplasma con más resistencia a sequía en $P$. vulgaris proviene de la raza Durango del acervo Mesoamericano (Terán y Singh 2002). Resultados con poblaciones interraciales han sugerido que el camino más efectivo es combinar alto rendimiento con resistencia de sequía dentro del acervo Mesoamericano (Singh, 1995; Terán y Singh, 2002). El genotipo ideal para sequía terminal es un genotipo que combine las características de resistencia a sequía de frijoles de la raza Durango con el arquetipo de los frijoles Mesoamericanos (Kelly et al. 1999). Este arquetipo se mejoró en la línea de MSU con resistencia a sequía, B98311, derivada de la cruza entre la variedad "Raven" de la raza Mesoamericana (Kelly et al. 1994) con la línea mejorada T-3016 con resistencia a sequía. T-3016 fue derivada de la cruza de Sierra/AC1028, y es una línea mejorada de la raza Durango de grano sin calidad comercial, identificada previamente como resistente a sequía en México y Michigan según la MG del rendimiento (Schneider et al. 1997). La línea B98311 fue seleccionada por ser un genotipo de alto rendimiento bajo sequía severa en Michigan en 1998 (Kolkman y Kelly 1999).

La obtención de alto rendimiento en frijol común bajo sequía terminal es limitada por el ataque de $M a$ crophomina phaseolina (Tassi) Goid, organismo causal de la enfermedad pudrición carbonosa. La pudrición carbonosa se ve favorecida por condiciones secas y resulta en la pérdida de hojas antes de madurez, reducción de vigor, y muerte de plantas en variedades susceptibles (Mayek-Pérez et al. 2002). Se observó una alta correlación entre la respuesta a la sequía y la susceptibilidad a $M$. phaseolina (Mayek-Pérez et al. 2001a). El genotipo TLP 19 fue el más resistente en un grupo diferencial creado para distinguir diferencias entre aislamientos de M. phaseolina de México (MayekPérez et al. 2001b), y se puede utilizar para la producción en regiones del trópico bajo, donde ocurre la enfermedad.

Los objetivos de este estudio fueron: identificar genotipos con resistencia a sequía en dos poblaciones de líneas recombinantes de frijol negro establecidas bajo condiciones de sequía terminal y riego en América Central y México; determinar la reacción de las líneas a la pudrición carbonosa y determinar la adaptación de las mejores líneas en el estado de Michigan.

\section{MATERIALES Y MÉTODOS}

Se cruzaron tres genotipos de frijol negro, B98311, TLP 19 y VAX 5, para desarrollar dos poblaciones de líneas recombinantes (RILs) para combinar la resistencia a sequía con calidad de semilla comercial de frijol negro. El genotipo con resistencia a sequía B98311 del programa de mejoramiento de la Universidad Estatal de Michigan (MSU), posee hábito de crecimiento tipo II y una raíz profunda y vigorosa (Frahm et al. 2003). La línea TLP 19 fue desarrollada para adaptación a suelos de bajo fósforo en el Centro Internacional de Agricultura Tropical (CIAT). Los genotipos fósforo-eficiente de frijol tienen un sistema de raíces poco profundo que se extiende superficialmente por el suelo con limitadas raíces basales (Rubio et al. 2003). La arquitectura de raíces contrastantes de B98311 y TLP 19 fue la consideración principal en su selección como progenitores, para crear genotipos con diferente sistemas de raíces 
que puedan contribuir en la resistencia a sequía. Bajo condiciones de sequía terminal en México, TLP 19 ha mostrado resistencia a la pudrición carbonosa (MayekPérez et al. 2001a). El tercer progenitor, VAX 5, se desarrolló en el CIAT de hibridaciones interespecíficas de frijol común y tépari (P. acutifolius A. Gray); esta línea fue seleccionada por su resistencia a la bacteriosis común (CBB) causada por Xanthomonas axonopodis p.v. phaseoli (Singh y Muñoz 1999). TLP 19 y VAX 5 se seleccionaron como progenitores por su adaptación a los trópicos bajos; y B98311 por su tolerancia a sequía y adaptación a condiciones templadas. Algunas características adicionales tales como tipo de semilla comercial, hábito de crecimiento erecto y resistencia a enfermedades se consideraron en la selección de los progenitores, a fin de asegurar el uso de los genotipos negros superiores que resultaran de este estudio en las regiones de América Central y México. Las cruzas originales se hicieron en 1998; al cruzar B98311/TLP 19 y B98311/VAX 5 se originaron las poblaciones L88 y L91, respectivamente. Para acelerar la obtención de RILs se utilizó el método descendencia de semilla individual en ambas poblaciones.

El 23 de enero del 2001, se sembraron 150 RILs, tres padres y siete testigos en Zamorano, Honduras $\left(14^{\circ} 00^{\prime} \mathrm{N}, 87^{\circ} 02^{\prime} \mathrm{W}, 800 \mathrm{~m}\right)$. Los siete testigos incluyeron el cultivar local de grano rojo Tío Canela-75, la línea mejorada EAP 9510-77, el cultivar Negro Tacaná (DOR 390) y el genotipo Negro 8025 de México, dos genotipos con resistencia a sequía BAT 477 y SEA 5 de CIAT, y el cultivar Río Tibagí de Brasil. Este experimento con 160 entradas se sembró como un diseño completamente al azar con tres repeticiones por cada condición de humedad, riego y sequía terminal. Las parcelas fueron de un surco individual de $5 \mathrm{~m}$ de longitud y $0,7 \mathrm{~m}$ de separación. Los tratamientos de sequía y riego se aplicaron a través del control del riego. Como el riego fue superficial, no se pudo cuantificar la cantidad de agua aplicada a cada tratamiento. Para cuantificar el rendimiento se cosecharon 30 plantas por surco. Se colectó información sobre la densidad de plantas y la incidencia de la pudrición carbonosa. La incidencia de la enfermedad (IE) se clasificó a los 75 días después de la siembra (DDS) como el número de plantas muertas en 50 plantas.

En Veracruz, México (18 44' N, 95 58' W, 16 m), las 150 RILs, dos padres (B98311 y TLP 19) y un testigo resistente a sequía (SEA 5) fueron sembrados en el Campo Experimental Cotaxtla el 24 de enero del 2002. El diseño experimental fue un factorial con cuatro tratamientos y dos repeticiones por cada tratamiento. Ambas poblaciones se expusieron a los tratamientos de sequía y riego. Se regó por surco y no se pudo determinar la cantidad de agua que se aplicó. Las parcelas fueron de $4 \mathrm{~m}$ de largo y 0,61 $\mathrm{m}$ de ancho y se cosechó una sección de 2,44 $\mathrm{m}^{2}$ para calcular el rendimiento. Un segundo tratamiento fue la inoculación con un aislamiento local de M. phaseolina para comparar con la infección natural del campo. Cada parcela se inoculó con 8 $\mathrm{g}$ de semilla de arroz colonizado con M. phaseolina. Se colectaron datos sobre densidad de plantas, rendimiento de grano y de pudrición carbonosa usando una escala de uno a nueve (Abawi y Pastor-Corrales 1990).

En el 2001, se estableció un ensayo de rendimiento de un grupo selecto de RILs y testigos locales en un ensayo de sequía de 11 entradas en Zamorano, Honduras, y un ensayo de 36 entradas con precipitación natural y riego en Montcalm, Michigan; y bajo condiciones de precipitación natural en un ensayo de 42 entradas en Saginaw, Michigan en el 2002. Las parcelas en Zamorano consistieron en surcos individuales de $5 \mathrm{~m}$ con cuatro repeticiones donde ambos, insectos y enfermedades, se controlaron con aplicaciones químicas y se aplicó riego ocho veces para un total de $200 \mathrm{~mm}$ ya que no llovió durante el experimento. Se colectaron datos sobre los días a inicio de floración y días a madurez. Las parcelas en Michigan consistieron en cuatro surcos (se incluyó un borde común de dos surcos), de 5,8 m de largo y 0,5 m de ancho. En el 2001, a los 7 DDS se inició una sequía donde la lluvia fue menor de cinco $\mathrm{mm}$ durante los siguientes treinta días. La lluvia fue de 255 mm durante una época de cuatro meses en el 2002 y no se aplicó riego. Para calcular el rendimiento se cosecharon $4,6 \mathrm{~m}^{2}$ de los dos surcos centrales.

Se realizó un análisis de varianza (ANDEVA) para cada experimento. Se usó PROC GLM en el experimento de El Zamorano con número de plantas cosechadas por parcela como covariable para ajustar el rendimiento (SAS Institute Inc. 2000). Se analizó cada tratamiento de sequía y riego en un diseño completamente al azar con tres repeticiones. Se calcularon las medias, diferencia mínima significativa (DMS) y valores de coeficientes de variación (CV) después del ajuste por la covariable. En Veracruz, se combinó los tratamientos inoculados y sin inocular con cuatro repeticiones dentro de los tratamientos de riego y sequía. Se analizó cada población separadamente en un diseño completamente al azar. El número de plantas fue usado como covariable para ajustar el rendimiento por parcela. El rendimiento promedio para cada RILs en el tratamiento bajo sequía, se usó con los rendimientos promedios correspondientes del tratamiento de riego para calcular la MG. El índice de intensidad de sequía [IIS $=1-(\mathrm{Rms} / \mathrm{Rmr})]$ se calculó para cada experimento, donde $\mathrm{Rms}$ es el rendimiento medio bajo sequía y Rmr es el rendimiento medio bajo riego para cada experimento (Fischer y Maurer 1978). Los valores de IIS representaron porcentajes de reducción del rendimiento 
por sequía, con el valor de 1 indicando la pérdida total del rendimiento y 0 indicando que no hubo efecto por sequía.

\section{RESULTADOS Y DISCUSIÓN}

En el experimento de Zamorano ocurrió una sequía terminal severa, IIS $=0,82$. Esta sequía fue más severa que en el experimento de Veracruz (IIS $=0,48$ ) y que en experimentos conducidos con frijol bajo condiciones de temporal en el altiplano semiárido de México (IIS = 0,49; Schneider et al. 1997) y bajo tratamiento de sequía bajo un cobertizo contra la lluvia en Michigan (IIS = 0,63; Ramírez-Vallejo y Kelly 1998). El desafío en el mejoramiento para resistencia a sequía es reproducir las condiciones de sequía consistentes entre experimentos, lo que puede realizarse sólo con la selección cuidadosa de los sitios de prueba. Los sitios de prueba en Zamorano y Veracruz se seleccionaron porque la sequía terminal natural es un acontecimiento que reduce la producción de frijol. Los programas locales de fitomejoramiento deben invertir en experimentos grandes con tratamientos contrastes de humedades para identificar cultivares de frijol con resistencia a sequía. Los experimentos de sequía resultan ser el doble en tamaño y los recursos son limitados; por lo que, los programas pequeños de mejoramiento solo evalúan el tratamiento de sequía o limitan el número de genotipos. Debido a que el rendimiento en riego $(\mathrm{Rr})$ tiene más influencia en la $\mathrm{MG}$ que el Rs, se sugiere estimar primero el rendimiento potencial y sólo evaluar bajo sequía las líneas con alto Rr. En este estudio, la respuesta consistente de las mejores líneas a través de los diversos ambientes de temporal y riego, sugiere la funcionalidad de esta estrategia.
El rendimiento de las 150 RILs varió de 2 a 842 $\mathrm{kg} / \mathrm{ha}$ bajo condiciones de sequía en Zamorano; mientras que, el rendimiento en las condiciones de riego varió de 1.130 a $2.922 \mathrm{~kg} / \mathrm{ha}$ (Cuadro 1). En Veracruz, el rendimiento de las mismas 150 RILs en sequía varió de 210 a $1.365 \mathrm{~kg} / \mathrm{ha}$ y de 462 a $2.402 \mathrm{~kg} / \mathrm{ha}$ bajo condiciones de riego. Se registraron efectos significativos de los genotipos en ambas poblaciones para cada tratamiento en el experimento de Veracruz (Cuadro 2); mientras que, en el 2001, sólo hubo diferencias significativas entre genotipos en el tratamiento de sequía para la población L88 en Zamorano debido a un alto CV en esta localidad. Las variaciones en rendimiento en las poblaciones L88 y L91 puede ser el resultado de su composición genética. B98311 fue el progenitor común de ambas poblaciones; posee resistencia a sequía de la línea de mejorada de la raza Durango y las características de rendimiento de un cultivar de la raza Mesoamericana. TLP 19 también es de la raza Mesoamericana y VAX 5 es el producto de una hibridación interspecífica de $P$. vulgaris y $P$. acutifolius. Es probable que exista un efecto negativo ligado a los genes de $P$. acutifolius presente en VAX 5 que puede ser un factor determinante en el reducido rendimiento de la población L91, cuando se compara con la mayor compatibilidad en la población L88 que se derivó principalmente de un antecedente genético de Mesoamérica. El germoplasma de la raza Mesoamericana se reconoce como una fuente de genes para el mejoramiento en ambientes secos y húmedos en América Central (White et al. 1994). En Zamorano, muchas RILs de la población L88 superaron en rendimiento a los testigos locales y genotipos resistentes a sequía de diferentes acervos genéticos tales como Rio Tibagí, BAT 477, SEA 5 y Tío Canela 75 (Cuadro 3).

Cuadro 1. Escala de rendimientos y medias de poblaciones L88 y L91 establecidas bajo riego y sequía en El Zamorano, Honduras en 2001 y Veracruz, México en 2002.

\begin{tabular}{|c|c|c|c|c|c|c|c|c|}
\hline \multirow[b]{3}{*}{ Escala } & \multicolumn{4}{|c|}{ Población L88 } & \multicolumn{4}{|c|}{ Población L91 } \\
\hline & & \multirow[t]{2}{*}{ Zamorano } & \multicolumn{2}{|c|}{ Veracruz } & & \multirow[t]{2}{*}{ Zamorano } & \multicolumn{2}{|c|}{ Veracruz } \\
\hline & & & $\begin{array}{l}\text { Inoculada } \dagger \\
- \text { kg/ha }--\end{array}$ & $\begin{array}{l}\text { Control } \\
-\end{array}$ & & & $\begin{array}{l}\text { Inoculada } \\
-\mathrm{kg} / \mathrm{ha}\end{array}$ & Control \\
\hline \multirow[t]{2}{*}{ Rs $\ddagger$} & $\max$ & 842 & 1.365 & 1120 & $\max$ & 599 & 775 & 854 \\
\hline & $\min$ & 77 & 465 & 332 & $\min$ & 2 & 210 & 265 \\
\hline Media & & $320(0,27) \S$ & $857(0,29)$ & $671(0,29)$ & & $207(0,20)$ & $442(0,22)$ & $533(0,20)$ \\
\hline \multirow[t]{2}{*}{$\mathrm{Rr}$} & $\max$ & 2.922 & 2.240 & 2.402 & $\max$ & 2.587 & 1.367 & 1.368 \\
\hline & $\min$ & 1.441 & 631 & 799 & $\min$ & 1.130 & 461 & 548 \\
\hline Media & & $2.057(0,04)$ & $1.430(0,16)$ & $1.624(0,15)$ & & $1.858(0,02)$ & $875(0,14)$ & $898(0,14)$ \\
\hline
\end{tabular}

$\dagger$ Inoculada con Macrophomina phaseolina; El control no se inoculó.

\$ Rs - Rendimiento en sequía, $\mathrm{Rr}$ - Rendimiento en riego.

$\S$ Media de incidencia de pudrición carbonosa por Macrophomina phaseolina entre paréntesis. 
Cuadro 2. Valores de cuadrados medios y nivel de significancia para fuentes de variación en poblaciones L88 y L91 establecidas en El Zamorano, Honduras y Veracruz, México en 2001 y 2002.

\begin{tabular}{|c|c|c|c|c|c|c|}
\hline \multirow{2}{*}{$\begin{array}{l}\text { Pob. L88 } \\
\text { Fuente }\end{array}$} & \multicolumn{3}{|c|}{ Zamorano, 2001} & \multicolumn{3}{|c|}{ Veracruz, 2002} \\
\hline & $\overline{\mathbf{G L} \dagger}$ & Ts & $\mathbf{T r}$ & GL & Ts & $\operatorname{Tr}$ \\
\hline Repetición & 2 & 102.574 & 755.201 & 3 & $1.471 .137 * * * *$ & $1.416 .776 * * * *$ \\
\hline Genotipo & 80 & $72.989 *$ & 304.003 & 80 & $72.891 * * * *$ & $308.982 * * * *$ \\
\hline Densidad & 1 & $840.106 * * * *$ & 461.861 & 1 & $1.607 .054 * * * *$ & $459.439 *$ \\
\hline Error & 159 & 49.863 & 320.694 & 239 & 31.041 & 96.838 \\
\hline C.V. (\%) & & 70,2 & 27.6 & & 22,9 & 20,2 \\
\hline
\end{tabular}

\begin{tabular}{|c|c|c|c|c|c|c|}
\hline \multirow{2}{*}{$\begin{array}{l}\text { Pob. L91 } \\
\text { Fuente }\end{array}$} & \multicolumn{3}{|c|}{ Zamorano, 2001} & \multicolumn{3}{|c|}{ Veracruz, 2002} \\
\hline & $\overline{\mathbf{G L}}$ & Ts & $\operatorname{Tr}$ & $\overline{\text { GL }}$ & Ts & Tr \\
\hline Repetición & 2 & 96.906 & 654.273 & 3 & $247.125 * * * *$ & 35.533 \\
\hline Genotipo & 68 & 49.461 & 357.196 & 68 & $53.978 * * * *$ & $160.596 * * * *$ \\
\hline Densidad & 1 & $158.990 *$ & 682.311 & 1 & $619.994 * * * *$ & $1.639 .751 * * * *$ \\
\hline Error & 135 & 35.870 & 330.826 & 203 & 14.429 & 30.122 \\
\hline C.V. $(\%)$ & 90,4 & 3,9 & & & 24,8 & 19,7 \\
\hline
\end{tabular}

$†$ GL - grados de libertad, CV - coeficiente de variación, Ts - Tratamiento de sequía, Tr Tratamiento de riego

$* \mathrm{P}<0,05, * * \mathrm{P}<0,01, * * * \mathrm{P}<0,001, * * * * \mathrm{P}<0,0001$.

La infestación de la pudrición carbonosa causó reducción en el número de plantas y mostró diferencias significativas en todos los tratamientos (Cuadro 2). Algunos genotipos pudieron compensar en rendimiento la pérdida de plantas. B98311, el padre resistente a sequía, sufrió reducción de plantas de $42 \%$ en Zamorano debi- do a la pudrición carbonosa, pero rindió más que los otros progenitores por más de $100 \mathrm{~kg} / \mathrm{ha}$ (Figura 1). En Veracruz, B98311 mostró una respuesta similar y exhibió amplia adaptación y estabilidad bajo diferente niveles de sequía; mientras que, TLP 19 obtuvo mayor rendimiento en la MG (1.046 kg/ha) que B98311 (991

Cuadro 3. Rendimiento de las RILs de mayor y menor rendimiento en la población L88, progenitores y testigos establecidos en El Zamorano, Honduras en 2001 y Veracruz, México en 2002.

\begin{tabular}{|c|c|c|c|c|c|c|}
\hline \multirow[b]{2}{*}{ Línea } & \multicolumn{3}{|c|}{ Honduras, 2001} & \multicolumn{3}{|c|}{ Veracruz, 2002} \\
\hline & MG $\dagger$ & Rs & $\mathbf{R r}$ & MG & Rs & $\mathbf{R r}$ \\
\hline L88-63 & 1.473 (1)末 & $842(1)$ & $2.576(6)$ & $1.468(1)$ & $1.036(2)$ & $2.080(3)$ \\
\hline L88-30 & $1.328(3)$ & $779(2)$ & $2.263(30)$ & $1.264(10)$ & $947(8)$ & $1.687(22)$ \\
\hline L88-69 & $1.286(4)$ & $680(4)$ & $2.432(18)$ & $1.120(34)$ & $711(60)$ & 1.765 (16) \\
\hline L88-13 & $1.285(5)$ & $565(9)$ & $2.922(1)$ & $1.182(27)$ & $873(18)$ & $1.600(37)$ \\
\hline L88-66 & $1.205(6)$ & $561(10)$ & $2.589(4)$ & $1.235(16)$ & 849 (24) & $1.797(12)$ \\
\hline L88-61 & $1.050(11)$ & 507 (13) & $2.173(39)$ & $1.329(5)$ & $839(25)$ & $2.105(2)$ \\
\hline L88-18 & $368(146)$ & $90(144)$ & $1.501(148)$ & $907(76)$ & $733(52)$ & $1.122(87)$ \\
\hline B98311(p) & $951(22)$ & $375(33)$ & $2.411(20)$ & $991(63)$ & $706(62)$ & $1.392(61)$ \\
\hline SEA 5 & $893(30)$ & $524(11)$ & $1.521(145)$ & $643(115)$ & $444(125)$ & 931 (110) \\
\hline Río Tibagi & $886(31)$ & $372(34)$ & $2.108(51)$ & - & - & - \\
\hline BAT 477 & $784(54)$ & $400(27)$ & $1.536(141)$ & - & - & - \\
\hline VAX 5 (p) & $663(90)$ & $249(82)$ & $1.765(110)$ & - & - & - \\
\hline TLP-19 (p) & $637(95)$ & 169 (118) & $2.399(22)$ & $1.046(55)$ & $756(44)$ & $1.446(56)$ \\
\hline Tío Canela 75 & $602(105)$ & $218(95)$ & 1.657 (129) & - & - & - \\
\hline Media & 699 & 275 & 1.517 & 1.077 & 764 & 1.527 \\
\hline DMS $(0,05)$ & & 333 & 933 & & 245 & 433 \\
\hline C.V. $(\%)$ & & 77 & 30 & & 23 & 20 \\
\hline
\end{tabular}

$†$ MG - Media geométrica, Rs - Rendimiento en sequía, Rr - Rendimiento en riego. \$ Posición en paréntesis, (p) designa progenitor; Media de 160 entradas en Zamorano y 153 entradas en Veracruz. 

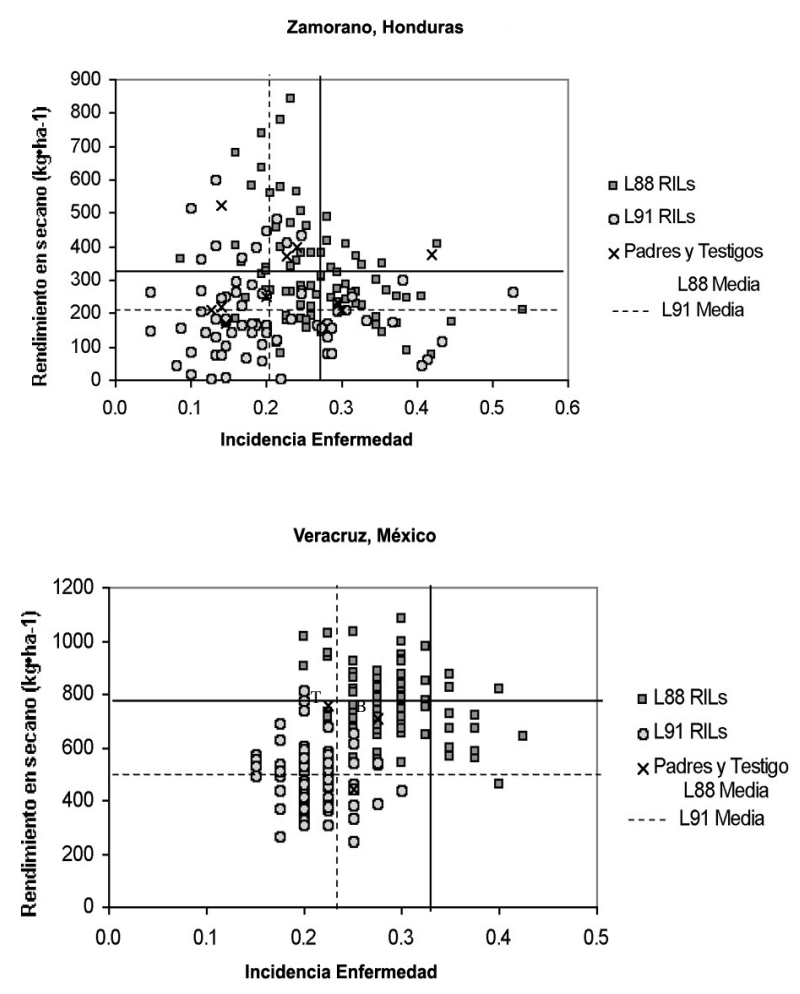

Figura 1. Gráfica de dispersión del rendimiento en sequía e incidencia de la pudrición carbonosa en 150 RILs de las poblaciones L88 y L91, se incluyen los progenitores B98311 (B), TLP 19 (T), VAX 5 (V) y testigos. Experimentos conducidos en El Zamorano, Honduras en 2001 y Veracruz, México en 2002.

$\mathrm{kg} / \mathrm{ha}$ ) mostrando su capacidad para rendir bajo sequía moderada. La población L88 mostró menor resistencia a la enfermedad $(0,27 ; 0,29)$ que la población L91 $(0,21 ; 0,21 ;$ Figura 1$)$. Con respecto a la presión de la pudrición carbonosa, la población L88 mostró mayor rendimiento en Zamorano y Veracruz (320 y $764 \mathrm{~kg} / \mathrm{ha}$ ) que la población L91 (207 y 491 kg/ha; Figura 1). Los genotipos sin un nivel suficiente de resistencia a la pudrición carbonosa no expresarán su potencial de rendimiento bajo sequía debido a los efectos negativos del patógeno, que resultan en pérdida de plantas y vigor y en consecuencia en rendimientos inferiores.

La MG proporciona la oportunidad de identificar las entradas de más alto rendimiento en una prueba individual y continuar con la evaluación de esos genotipos en más ensayos. Por ejemplo, L88-63 y L88-69 ocuparon las posiciones primera y cuarta en la MG del rendimiento en Zamorano y no fueron diferente en Rs o $\operatorname{Rr}$ (Cuadro 3). Cuando éstas se evaluaron en Saginaw, Michigan en el 2002, rindieron consistentemente alto y ocuparon las posiciones primera y segunda entre las RILs de esta población en el experimento, demostrando alto potencial de rendimiento. En Veracruz, L88-63 rindió consistentemente alto y ocupó la primera posición en la MG del rendimiento (Cuadro 3), mientras que L88-69 ocupó la trigésima cuarta posición en la MG del rendimiento (1120 kg/ha) entre las RILs de la población L88. A los genotipos como L88-63 que consistentemente mostró la posición más alta, sin considerar la significancia estadística, se les debe dar atención especial por parte de los mejoradores ya que pudieran poseer la combinación deseada de las características que son necesarias en ambientes con sequía. Un sistema de posiciones basado en el rendimiento favorecerá genotipos que puedan rendir por encima de la media aún con la presión de enfermedad característica de ambientes secos; una estrategia preferible sería incrementar el nivel de resistencia a M. phaseolina en esos genotipos con alta MG de los rendimientos. En experimentos subsiguientes, la severidad de la sequía deberá ser suficiente para inducir suficiente presión de enfermedad, ya que no hubo diferencias significativas en la incidencia de la enfermedad entre los tratamientos de inoculación y control en Veracruz (Cuadro 1).

Cuadro 4. Número de entradas, posición de L88-63 y rendimiento en porcentaje con relación a la media del sitio de prueba en cinco localidades diferentes en Honduras, México y Michigan durante 2001 y 2002.

\begin{tabular}{lrrrrrrrrr}
\hline \multicolumn{1}{c}{ Localidad y Año } & $\begin{array}{c}\text { Núm. de } \\
\text { entradas }\end{array}$ & \multicolumn{2}{c}{ MG $\dagger$} & & \multicolumn{2}{c}{ Rs } & \multicolumn{2}{l}{ Rr } \\
\hline Zamorano, Honduras 2001 & 160 & 1ra & $153 \%$ & 1 ra & $168 \%$ & 6 ta & $124 \%$ \\
Montcalm, Michigan 2001 & 36 & 3ra & $119 \%$ & 16 ta & $102 \%$ & 2 da & $130 \%$ \\
Veracruz, México 2002 & 153 & 1ra & $134 \%$ & 2 da & $133 \%$ & 3 ra & $136 \%$ \\
Saginaw, Michigan 2002 & 42 & - & - & - & - & 4 ta & $111 \%$ \\
Zamorano, Honduras 2002 & 11 & - & - & 1ra & $123 \%$ & - & - \\
\hline
\end{tabular}

$\dagger$ MG - Media geométrica, Rs - Rendimiento en secano, $\mathrm{Rr}$ - Rendimiento en riego. 
Una línea recombinante, la L88-63, ocupó la primera posición en la MG entre todas las líneas en Zamorano y en Veracruz y rindió $153 \%$ y $134 \%$ por encima de las medias de cada sitio, respectivamente (Cuadro 4). La selección de L88-63 se basó en la MG del rendimiento usando solo los datos de Zamorano 2001. Ensayos posteriores en el 2002, del potencial de las RILs con resistencia a sequía, dieron rendimientos medios contrastantes de $3667 \mathrm{~kg} / \mathrm{ha}$ y $871 \mathrm{~kg} / \mathrm{ha}$ en el ambiente húmedo de Saginaw, Michigan y bajo sequía en Zamorano, Honduras, respectivamente. A pesar de esa gran diferencia en rendimiento, la línea L88-63 ocupó la primera posición entre las RILs y testigos y rindió por encima de la media de cada sitio, en las localidades respectivas, por $111 \%$ y $123 \%$ (Cuadro 4). La superioridad de esta línea apoya la evaluación de la misma en otros ambientes donde la sequía terminal sea un problema.

\section{CONCLUSIONES}

El sistema de posición de la MG del rendimiento utilizado en este estudio permitió la identificación de L88-63, el cual es un genotipo de alta resistencia a sequía, con amplia adaptación en los trópicos bajos y en regiones templadas. A pesar de la complejidad del mejoramiento para resistencia a sequía en frijol común, la resistencia se transfirió efectivamente por métodos sencillos de cruzamiento y selección en localidades apropiadas de evaluación. El mejoramiento para sequía terminal en América Central y México requiere la incorporación de un mayor nivel de resistencia a $M$. phaseolina para alcanzar las necesidades del área de producción. La calidad comercial de semilla de L88-63 permitirá su utilización en el mejoramiento del frijol negro, el cual es una clase comercial de alta demanda en las regiones de los trópicos bajos de América Central y México.

\section{LITERATURA CITADA}

ABAWI, G.S.; PASTOR-CORRALES, M.A. 1990. Root rots of beans in Latin America and Africa: Diagnosis, research methodologies, and management strategies. CIAT, Cali, Colombia, 114 pp.

ABEBE, A.; BRICK, M.A.; KIRKBY, R.A. 1998. Comparison of selection indices to identify productive dry bean lines under diverse environmental conditions. Field Crops Res. 58: 15-23.

COTTY, D.; GARCÍA, M.; ESTRADA, I.; ANCHUNDIA, E. 2001. Indicadores básicos sobre el desempeño agrope- cuario 1971-2000. In: Moya, J., (Ed.), Proyecto de Información Agrícola y Análisis de Políticas. Instituto Nacional de Estadística, Zamorano, Honduras. 34-38 pp.

FISCHER, R.A.; MAURER, R. 1978. Drought resistance in spring wheat cultivars. I. Grain yield responses. Aust. J. Agr. Res. 29: 897-912.

FRAHM, M.A.; FOSTER, E.F.; KELLY, J.D. 2003. Indirect screening techniques for drought resistance in dry beans. Annu. Rept. Bean Improv. Coop. 46: 87-88.

HALL, A.E. 1993. Is dehydration tolerance relevant to genotypic differences in leaf senescence and crop adaptation to dry environments? In: Close, T.J., Bray, E.A. (Eds.) Plant response to cellular dehydration during environmental stress. The American Society of Plant Physiologists. 1-9 pp.

KELLY, J.D.; SCHNEIDER, K.A.; KOLKMAN, J.M. 1999. Developments in Plant Breeding: Breeding to improve yield. In: Singh, S.P. (ed.), Common bean improvement in the Twenty-First Century. Kluwer Acad. Pub., Dordrecht, The Netherlands. 187-222 pp.

KELLY, J.D.; HOSFIELD, G.L.; VARNER, G.V.; UEBERSAX, M.A.; HALEY, S.D.; TAYLOR J. 1994. Registration of "Raven" black bean. Crop Sci. 34: 14061407.

KOLKMAN, J.M.; KELLY, J.D. 1999. Drought stress and bean productivity during the 1998 growing season. Michigan Dry Bean Digest 23: 2-3.

MAYEK-PÉREZ, N.; LÓPEZ-CASTAÑEDA, C.; LÓPEZSALINAS, E.; ACOSTA-GALLEGOS, J.A. 2001a. Herencia de la resistencia genética a Macrophomina phaseolina (Tassi) Goid. en frijol. Agrociencia 35: 637648.

MAYEK-PÉREZ, N.; LÓPEZ-CASTAÑEDA, C.; GONZÁLEZ-CHAVIRA， M.; GARCÍA-ESPINOSA， R.; ACOSTA-GALLEGOS, J.; MARTÍNEZ DE LA VEGA, O.; SIMPSON, J. 2001b. Variability of Mexican isolates of Macrophomina phaseolina based on pathogenesis and AFLP genotype. Physiol. Mol. Plant P. 59: 257-264.

MAYEK-PÉREZ, N.; GARCÍA-ESPINOSA, R.; LÓPEZCASTAÑEDA, C.; ACOSTA-GALLEGOS, J.; SIMPSON, J. 2002. Water relations, histopathology and growth of common bean (Phaseolus vulgaris L.) during pathogenesis of Macrophomina phaseolina under drought stress. Physiol. Mol. Plant P. 60: 185-195. 
RAMIREZ-VALLEJO, P.; KELLY, J.D. 1998. Traits related to drought resistance in common bean. Euphytica 99: 127-136.

ROSAS, J.C.; ERAZO, J.D.; MONCADA, J.R. 1991. Tolerancia a la sequía en germoplasma de frijol común y frijol tepari. CEIBA 32: 91-105.

RUBIO, G.; LIAO, H.; YAN, X.; LYNCH, J.P. 2003. Topsoil foraging and its role in plant competitiveness for phosphorus in common bean. Crop Sci. 43: 598-607.

SAMPER, C. 1984. Effects of water stress imposed at midpod filling upon yield and dry matter partitioning in dry beans (Phaseolus vulgaris L.). Tesis MSc., East Lansing, MI, EE.UU., Universidad Estatal de Michigan. $131 \mathrm{p}$.

SAS INSTITUTE INC. 2000. SAS, Version 8.0, SAS Institute Inc., Cary, NC. 3884 p.
SCHNEIDER, K.A.; ROSALES-SERNA, R.; IBARRA-PEREZ, F.; CAZARES-ENRIQUEZ, B.; ACOSTA-GALLEGOS, J.A.; RAMIREZ-VALLEJO, P.; WASSIMI, N.; KELLY, J.D. 1997. Improving common bean performance under drought stress. Crop Sci. 37: 43-50.

SINGH, S.P. 1995. Selection for water-stress tolerance in interracial populations of common bean. Crop Sci. 35: 118-124.

SINGH, S.P.; MUÑOZ, C.G. 1999. Resistance to common bacterial blight among Phaseolus species and common bean improvement. Crop Sci. 39: 80-89.

TERÁN, H.; SINGH, S.P. 2002. Comparison of sources and lines selected for drought resistance in common bean. Crop Sci. 42: 64-70.

WHITE, J.W.; OCHOA-M., R.; IBARRA-PEREZ., F.; SINGH, S.P. 1994. Inheritance of seed yield, maturity and seed weight of common bean (Phaseolus vulgaris) under semi-arid rainfed conditions. J. Agr. Sci. 122: 265-273. 